\title{
Avaliação clínico-radiográfica da mobilidade da lordose lombar*
}

\author{
Radiographic and clinical mobility \\ evaluation in lumbar lordosis
}

\author{
Antônio Vítor de Abreu', Alexandre Peixoto de Mello², \\ Gustavo Silva Trovão², CÉsar Rubens da Costa Fontenelle ${ }^{3}$
}

\section{RESUMO}

O objetivo deste trabalho foi estabelecer o padrão normal dos valores angulares para a lordose lombar, determinar a mobilidade clínico-radiológica no plano sagital (flexo-extensão) da coluna lombar e testar a força dos músculos abdominais em indivíduos de ambos os sexos em três faixas etárias. Métodos: Foram avaliados 150 indivíduos, sem queixas álgicas ou deformidades vertebrais, todos escolhidos aleatoriamente. Os participantes foram divididos em três grupos de 50 indivíduos de acordo com a faixa etária - I) 0 a 20; II) 21 a 40 e III) 41

* Trabalho realizado no Serviço de Traumato-Ortopedia do Hospital Universitário Clementino Fraga Filho da Universidade Federal do Rio de Janeiro - UFRJ - Rio de Janeiro (RJ), Brasil.

1. Professor Associado do Departamento de Ortopedia e Traumatologia da Faculdade de Medicina da Universidade Federal do Rio de Janeiro - UFRJ - Rio de Janeiro (RJ), Brasil. Chefe do Serviço de Traumato-Ortopedia do HUCFF/UFRJ, Brasil.

2. Mestre em Ortopedia e Traumatologia da Universidade Federal do Rio de Janeiro - UFRJ - Rio de Janeiro (RJ), Brasil.

3. Médico Ortopedista e Chefe de Clínica do Serviço de TraumatoOrtopedia do Hospital Universitário Clementino Fraga Filho da Universidade Federal do Rio de Janeiro - UFRJ - Rio de Janeiro (RJ), Brasil.

Endereço para correspondência: Rua Otaviano Hudson, 25, cob. 1.101, Copacabana - 22030-030 - Rio de Janeiro (RJ), Brasil.

E-mail: mfpinto16@terra.com.br

Recebido em 24/1/06. Aprovado para publicação em 16/10/07. Copyright RBO2007 a 60 - totalizando 79 do sexo masculino e 71 do feminino. Todos foram submetidos a exames radiográficos da coluna lombar em ortostatismo, na incidência em perfil, com o tronco em flexão máxima e em posição neutra. Os indivíduos da pesquisa foram também avaliados quanto à capacidade de realizar exercícios abdominais com os membros inferiores estendidos e fletidos, para investigar a atuação dos músculos lordogênicos e antilordogênicos, respectivamente. A partir das radiografias, foram medidos os seguintes ângulos: a) entre o topo de L1 e o topo de L5; b) topo de L1 e a base de L5; c) topo de L1 e o topo de S1; d) base de L5 e o topo de S1 (ângulo da articulação lombossacra) e e) topo de L5 e base de L5 (acunhamento de L5). Resultados: Nos três grupos, os resultados médios para os ângulos a, b e c foram, respectivamente, de $37^{\circ}$, $44^{\circ}$ e $57^{\circ}$ em neutro e de $-1^{\circ}, 6^{\circ}$ e $11^{\circ}$ em flexão máxima. No grupo I, as médias foram de $33,3^{\circ}, \mathbf{4 2}^{\circ}$ e $56,7^{\circ}$ em neutro e de $-18,8^{\circ},-12,4^{\circ}$ e $-12,4^{\circ}$ em flexão máxima; no grupo II, foram de $36,1^{\circ}, 42,9^{\circ} \mathrm{e}$ $55,9^{\circ}$ em neutro e de $4,2^{\circ}, 10,6^{\circ}$ e $18,1^{\circ}$ em flexão máxima; e no grupo III, de $41,2^{\circ}, 48^{\circ}$ e $58,2^{\circ}$ em neutro e de $12,6^{\circ}, 18,5^{\circ}$ e $26,5^{\circ}$ em flexão máxima. Para o ângulo da articulação lombossacra, as médias foram de $14,6^{\circ}, 12,2^{\circ}$ e $10,1^{\circ}$ em neutro e de $1,4^{\circ}, 7,5^{\circ}$ e $7,9^{\circ}$ em flexão máxima, para os grupos I, II e III, respectivamente. A média do ângulo de acunhamento de $L 5$ foi de $8,6^{\circ}, 7,6^{\circ}$ e $4,4^{\circ}$ em neutro e de $5,9^{\circ}, 6,1^{\circ}$ e $3,6^{\circ}$ em flexão, para os grupos I, 
II e III, respectivamente. Exercício abdominal com os membros inferiores estendidos foi realizado por $100 \%$ dos indivíduos no grupo I, $70 \%$ no II e $48 \%$ no III, enquanto que com os membros inferiores fletidos foi executado por $96 \%$ do grupo I, $46 \%$ do II e $16 \%$ do III. Conclusão: Decrescem concomitantemente a mobilidade em flexão da coluna lombossacra e a força dos músculos abdominais com $o$ aumento da idade.

Descritores - Lordose/radiografia; Coluna vertebral/radiografia; Diagnóstico por imagem; Dor lombar/ diagnóstico

\section{ABSTRACT}

The objective of this paper was to establish a normal standard for the values of angles for lumbar lordosis, to determine the clinical and radiological mobility in the sagittal (flexion-extension) of the lumbar spine and to test the strength of abdominal muscles in individuals of both genders and three different age brackets. Methods: 150 individuals who had no pain complaints or vertebral deformities were evaluated, all of them chosen at random. The participants were divided into three groups of 50 individuals according to the age bracket - I) 0 to 20; II) 21 to 40, and III) 41 to 60 - in a total of 79 males and 71 females. All of them were submitted to radiographic exams of the lumbar spine in orthostatism, in profile, with the trunk in maximum flexion and in the neutral position. The research subjects were also evaluated for their capacity of performing abdominal exercises with extended and flectioned lower limbs in order to investigate the performance of lordogenic and anti-lordogenic muscles, respectively. On the X-rays, the following angles were measured: a) between the top of $L 1$ and the top of L5; b) top of L1 and base of L5; c) top of L1 and top of S1; d) base of L5 and top of S1 (angle of the lumbosacral joint) and e) top of L5 and base of L5 (wedging of de L5). Results: In the three groups, the mean results for angles $a, b$ and $c$ were, respectively, $37^{\circ}, 44^{\circ}$, and $57^{\circ}$ in neutral position, and $-1^{\circ}, 6^{\circ}$, and $11^{\circ}$ in maximum flexion. In group $I$, the means were $33.3^{\circ}, 42^{\circ}$, and $56.7^{\circ}$, in neutral position, and $-18.8^{\circ}$, $-12.4^{\circ}$, and $-12.4^{\circ}$ in maximum flexion; in group II, they were $36.1^{\circ}, 42.9^{\circ}$, and $55,9^{\circ}$ in neutral position, and $4.2^{\circ}, 10.6^{\circ}$, and $18.1^{\circ}$ in maximum flexion, and in group III, $41.2^{\circ}, 48^{\circ}$, and $58.2^{\circ}$, in neutral position, and $12.6^{\circ}, 18.5^{\circ}$, and $26.5^{\circ}$ in maximum flexion. For the lumbosacral joint angle, means were $14.6^{\circ}, 12.2^{\circ}$, and $10.1^{\circ}$ in neutral position, and $1.4^{\circ}, 7.5^{\circ}$, and $7.9^{\circ}$ in maximum flexion for groups I, II, and III, respectively. The mean of the wedging angle of $L 5$ was of $8.6^{\circ}, 7.6^{\circ}$, and $4.4^{\circ}$ in neutral position, and $5.9^{\circ}$, $6.1^{\circ}$, and $3.6^{\circ}$ in flexion, for groups I, II, and III, respectively. Abdominal exercise with extended lower limbs was performed by $100 \%$ of the individuals in group I, 70\% in II, and 48\% in III; and with flectioned lower limbs it was performed by $96 \%$ in group I, $46 \%$ in II, and 16\% in III. Conclusion: Increase in age is followed by a concomitant decrease in the flexion mobility of the lumbosacral spine and of the strength in abdominal muscles.

Keywords - Lordosis/radiography; Spine/radiography; Imaging diagnostic; Low back pain/diagnosis

\section{INTRODUÇÃO}

A incidência de dor lombar na população em geral é extremamente elevada. As causas do aparecimento dos sintomas são muitas, o que justifica a dificuldade no diagnóstico etiológico. Lombalgias são freqüentes na idade adulta e na velhice, sendo mais raras na infância e adolescência. $\mathrm{O}$ conhecimento da anatomia da coluna e de sua fisiologia é de grande importância para se compreender a etiopatogenia da dor lombar.

Além das vértebras e dos músculos, a coluna depende do funcionamento dos discos intervertebrais e dos ligamentos, tanto para a sustentação quanto para a mobilização. A carga que a coluna lombar suporta está relacionada ao alinhamento da coluna.

Com o envelhecimento, ocorrem perda da mobilidade das articulações da coluna vertebral, diminuição na potência muscular e hipotrofia dos músculos abdominais, com o predomínio do iliopsoas e paravertebrais lombares sobre a musculatura ântero-lateral do abdome. Isso provoca estruturação dos componentes 
da coluna lombar na posição de hiperlordose, configurando hiperextensão do conjunto lombossacro.

Na prática clínica diária, observa-se que o grau de lordose lombar é variável durante a vida. Normalmente, existe boa mobilidade lombar na infância e adolescência, enquanto na idade adulta, com o aparecimento de fatores de risco como a gravidez, o sedentarismo, a obesidade e a osteoporose, a coluna lombar apresenta redução da mobilidade. A ação dos músculos iliopsoas, na idade adulta, passa a exercer flexão da pelve sobre os quadris, substituindo a ação dos abdominais. Assim sendo, músculos essencialmente flexores da coxa sobre a pelve aumentam sua força, quando comparados com os abdominais, configurando o desequilíbrio entre os músculos lordogênicos e antilordogêni$\cos$.

A identificação do momento em que esse desequilíbrio se estabelece deverá ser fator decisivo na profilaxia da estruturação da coluna lombar em hiperextensão, ou seja, em hiperlordose. Essa perda da mobilidade da coluna lombar em razão do desequilíbrio muscular pode ser causa de aumento da pressão nas articulações interfacetárias, que levaria à artrose, sendo reconhecida como síndrome facetária. A profilaxia do desequilíbrio muscular lombar é, em última análise, elemento fundamental na redução dos índices de lombalgia na população em geral.

O objetivo do presente estudo foi demonstrar a perda da mobilidade da coluna lombar, bem como a estruturação em hiperlordose, por meio da análise, em três diferentes faixas etárias, do valor angular da lordose, da amplitude máxima de flexão da coluna lombar e da suficiência da musculatura abdominal. A partir dessa observação, poderão ser sugeridos programas de prevenção da estruturação da hiperlordose lombar e suas consequiências, como lombalgia secundária à evolução da síndrome facetária.

\section{MÉTODOS}

A casuística constou de 150 indivíduos, selecionados ao acaso nos Ambulatórios do Serviço de Traumato-Ortopedia do Hospital Universitário Clementino Fraga Filho da Universidade Federal do Rio de Janeiro (STO-HUCFF/UFRJ), utilizando-se como critérios de exclusão a queixa de dor vertebral, deformidades ou malformações vertebrais.

Os participantes foram informados sobre os detalhes da pesquisa, sendo solicitada autorização para que o exame radiográfico fosse realizado mediante assinatura de um termo de consentimento. Os seguintes dados pessoais eram colhidos para caracterizar a população pesquisada: nome, idade, sexo, peso corporal e altura. Não foram pesquisados os dados étnicos neste estudo.

Os indivíduos, após identificação e levantamento dos dados, eram examinados mediante da inspeção do tronco para a pesquisa de eventuais deformidades ou malformações.

Os grupos eram formados por indivíduos de ambos os sexos e apresentavam distribuição homogênea, sem predomínio de nenhum dos sexos. O grupo I foi constituído de 29 indivíduos do sexo masculino (58\%) e 21 do feminino (42\%). O grupo II apresentou igualdade, com 25 indivíduos do sexo masculino (50\%) e 25 do feminino (50\%). O grupo III teve 26 indivíduos do sexo masculino (52\%) e 24 do feminino (48\%).

A média de altura foi de $1,37 \mathrm{~m}$ no grupo I, $1,70 \mathrm{~m}$ no grupo II e 1,69m no grupo III. O peso médio foi de $38,9 \mathrm{~kg}$ no grupo I, $71,2 \mathrm{~kg}$ no grupo II e $70,9 \mathrm{~kg}$ no grupo III.

Foi solicitada a todos os participantes a execução de testes para a avaliação da musculatura abdominal; dois tipos de teste foram realizados. No primeiro, o indivíduo, deitado na maca, deveria fazer uma flexão do tronco sobre os membros inferiores estendidos, com os pés fixados à maca pelo examinador e as mãos na nuca (figura 1). $\mathrm{O}$ segundo diferia apenas na posição dos membros inferiores, que ficavam fletidos (figura 2). $\mathrm{O}$ teste era considerado positivo quando era realizada ao menos uma repetição do exercício. Os exercícios abdominais feitos com os quadris e joelhos estendidos avaliavam a força total de flexão do tronco - quando se tem substancial ajuda dos iliopsoas - enquanto naqueles com os quadris e joelhos fletidos anulavam-se os flexores dos quadris e isolavam-se os retos e oblíquos (figuras 1 e 2).

Teste I $\rightarrow$ avalia a capacidade de realizar a flexão do tronco na posição supina com os quadris e os joe- 

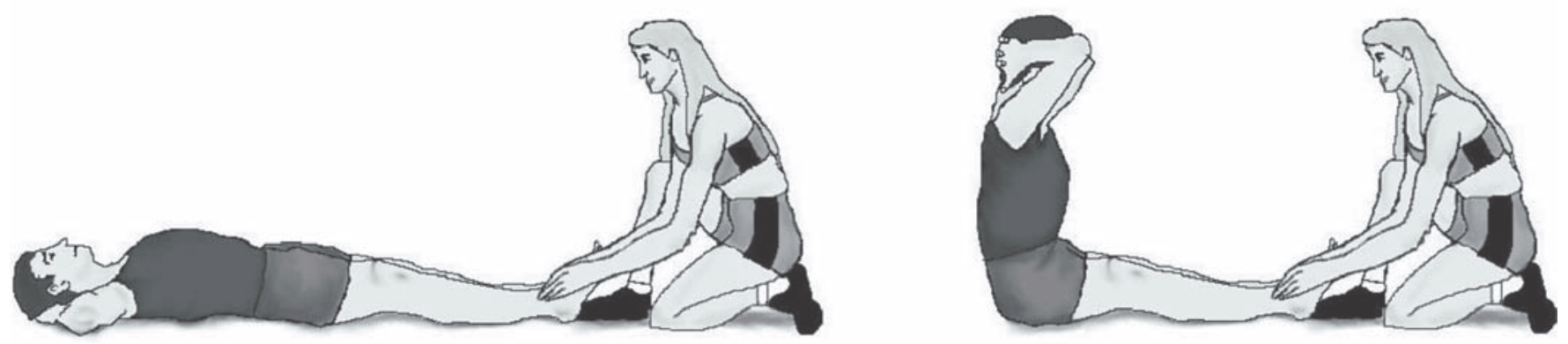

Figura 1 - Teste I: Abdominal com os membros inferiores estendidos.
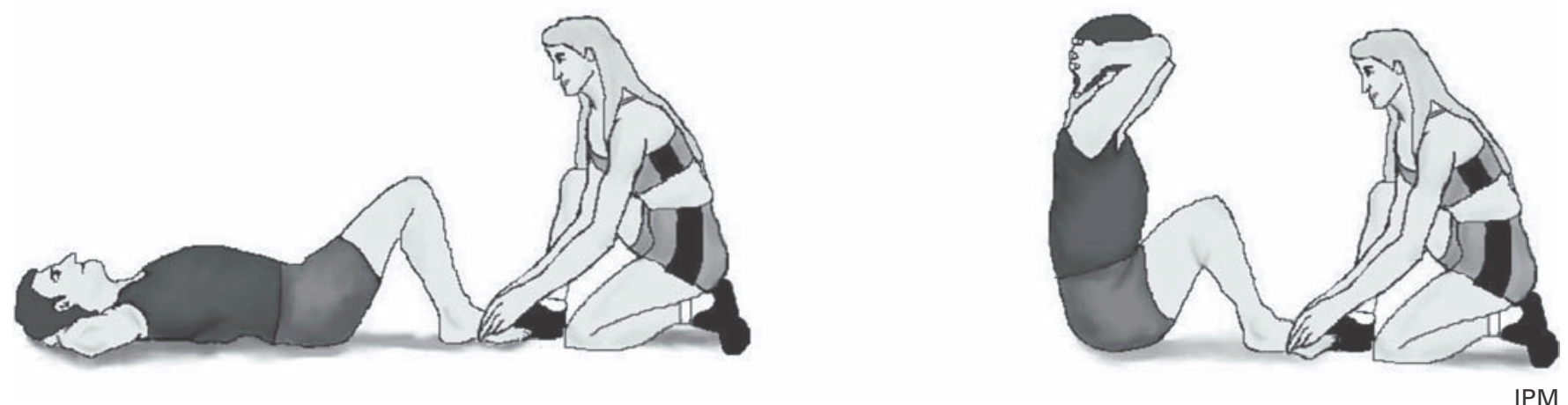

Figura 2 - Teste II: Abdominal com os membros inferiores fletidos.

lhos estendidos. Predominam nessa ação os músculos iliopsoas (figura 1).

Teste II $\rightarrow$ avalia a capacidade de realizar a flexão do tronco na posição supina com os quadris e os joelhos fletidos. Predominam nessa ação os músculos retos abdominais e oblíquos, anulando-se, com a flexão dos quadris e joelhos, a ação dos iliopsoas (figura 2).

As radiografias foram realizadas na posição ortostática em três incidências: ântero-posterior, perfil em neutro (figura 3) e perfil em flexão máxima (figura 4) da coluna lombar.

A avaliação radiográfica da coluna lombossacra era feita medindo-se cinco ângulos em cada paciente na posição ortostática em perfil, tanto em neutro quanto em flexão máxima. Consideraram-se os ângulos positivos quando em lordose e negativos quando em cifose.

As radiografias em ântero-posterior (AP) permitiram identificar desvios (escoliose), número de vértebras lombares e integridade discal.

Nas radiografias em perfil foram traçadas quatro linhas (figura 5): $1^{\mathrm{a}}-$ na borda superior de L1; $2^{\mathrm{a}}-$ na

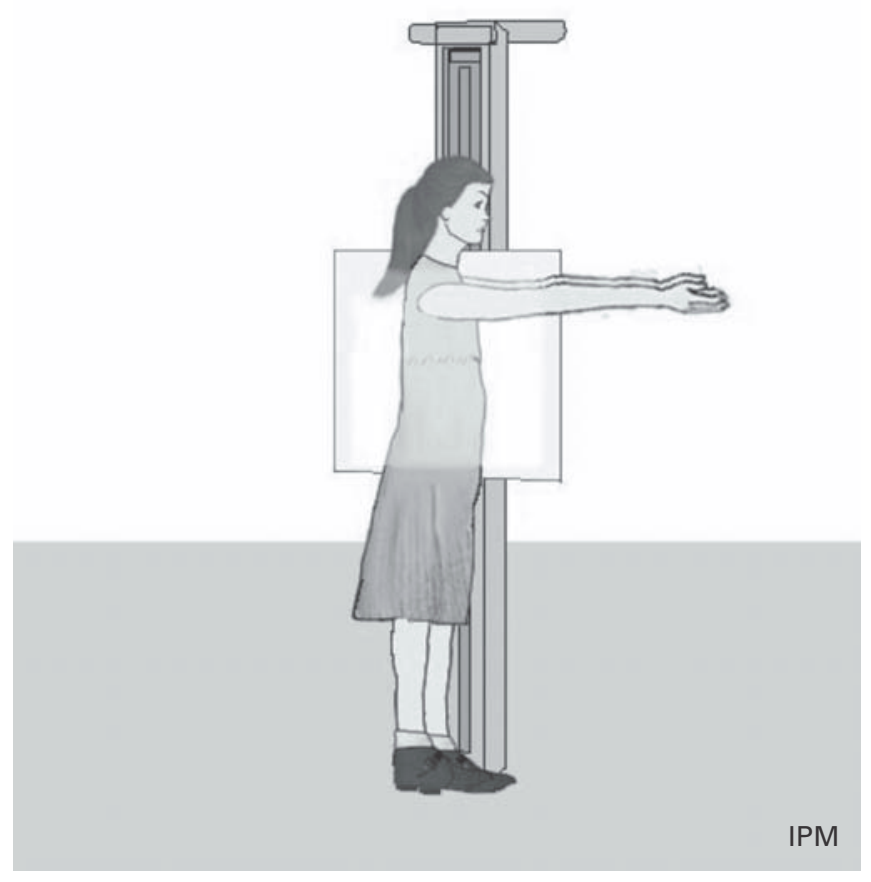

Figura 3 - Posição do paciente em perfil em neutro para ser radiografado 


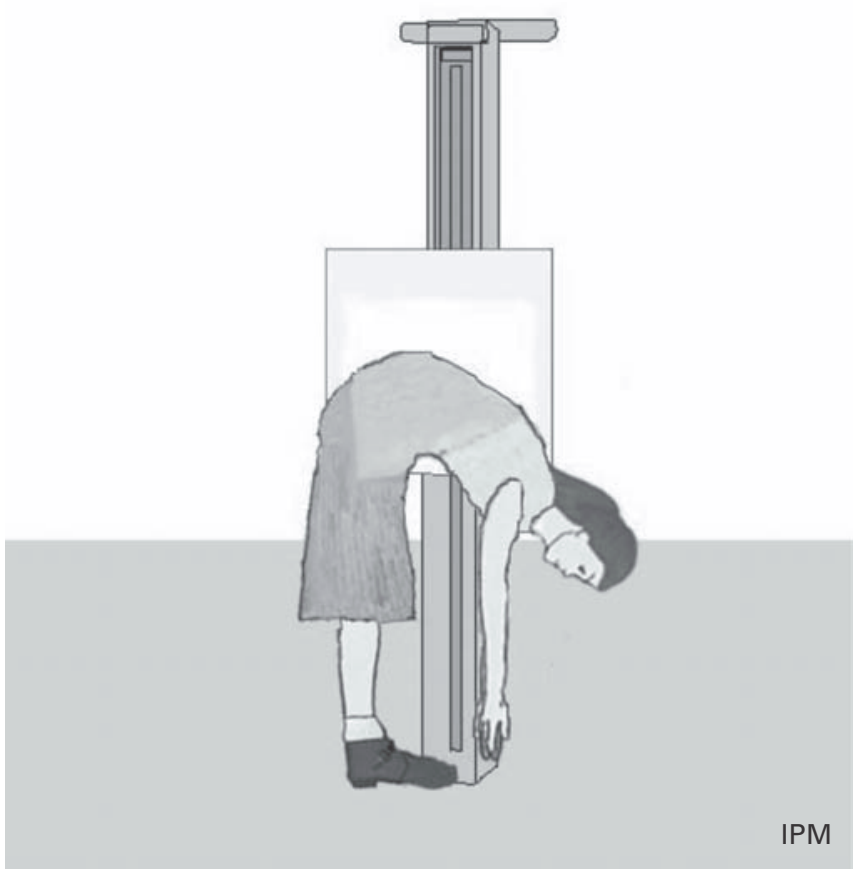

Figura 4 - Posição do paciente em perfil com flexão para ser radiografado

borda superior de L5; $3^{\mathrm{a}}$ - na borda inferior de L5; 4 na borda superior de $\mathrm{S} 1$.

A partir dessas linhas determinaram-se os seguintes ângulos (figura 6):

A1 - ângulo da lordose lombar (Propst-Proctor e Bleck $\left.^{(1)}\right)$ : entre a $1 \underline{\text { a e a } 3 \text { a }}$.

A2 - ângulo da lordose lombar (Wiltse e Winter ${ }^{(2)}$ ): entre a $1^{\mathrm{a}}$ e a 2 .

A3 - ângulo da lordose lombossacra ou lordose total: entre a $1^{\mathrm{a}}$ e a $4^{\mathrm{a}}$.

A4 - ângulo da articulação lombossacra: entre a 3르 e a 4 a

A5 - ângulo da cunha de L5: entre a 2 ${ }^{\mathrm{a}}$ e a 3a .

Os dados foram agrupados segundo a faixa etária da seguinte forma: grupo I - indivíduos de 1 a 20 anos; grupo II - indivíduos de 21 a 40 anos; e grupo III indivíduos de 41 a 60 anos.

Cada grupo era subdividido em relação ao sexo.

De cada grupo assim formado extraíram-se a média aritmética e o desvio-padrão. Essas medidas foram feitas em relação aos grupos e aos subgrupos. Foi

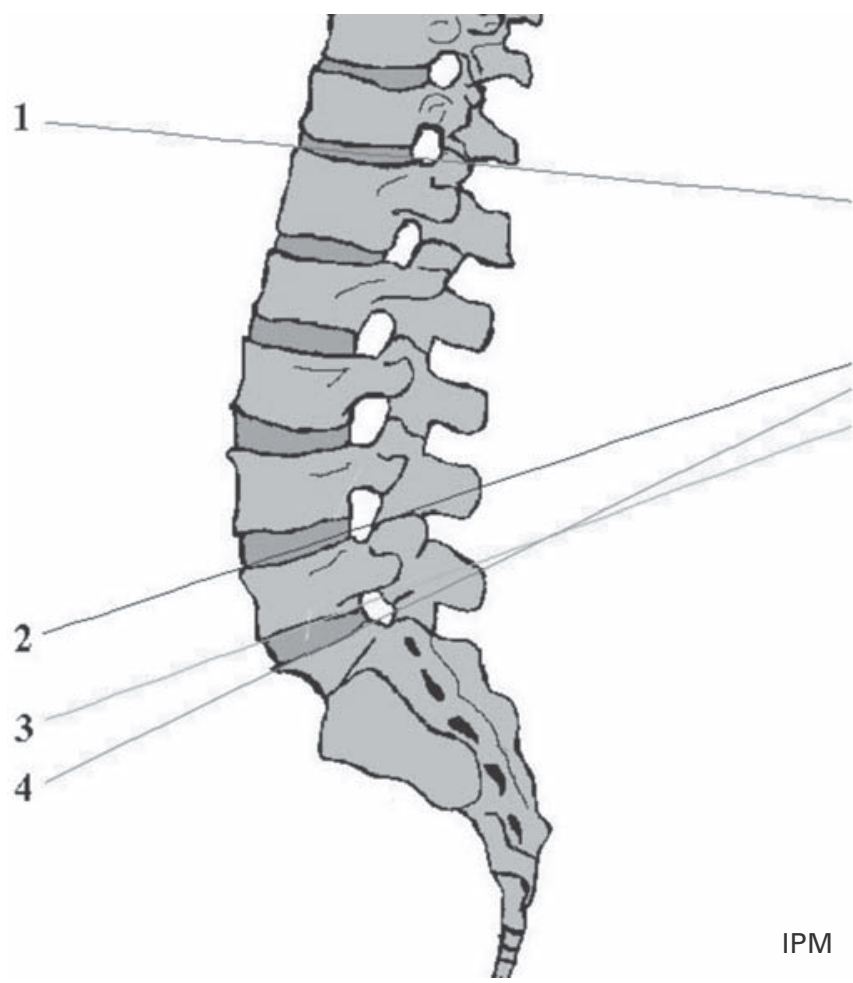

Figura 5 - Representação esquemática da coluna lombossacra em perfil, com suas respectivas linhas

medida ainda a mobilidade lombar, que é a diferença entre as medidas em neutro e em flexão.

Foi feita a comparação entre os grupos e, em cada grupo, entre os subgrupos, utilizando-se o teste $t$ de Student, tendo sido considerados significativos valores iguais ou inferiores a 0,15 .

\section{RESULTADOS}

Todos os resultados desta pesquisa estão resumidos na tabela 1. Para melhor visualização dos resultados foram colocados gráficos para ilustrar os achados de cada medição. Foi utilizada a expressão estatisticamente significante para aqueles testes $t$ de Student que retornaram valores menores ou iguais a 0,15 .

No grupo I o teste abdominal I foi realizado em $100 \%$ (dos homens e mulheres), enquanto o teste II foi feito por $96 \%$ dos indivíduos $(100 \%$ dos homens e $90 \%$ das mulheres). No grupo II o teste I foi realizado com sucesso por $70 \%$ dos indivíduos $(68 \%$ dos homens e $72 \%$ das mulheres) e o teste II, por $46 \%$ 


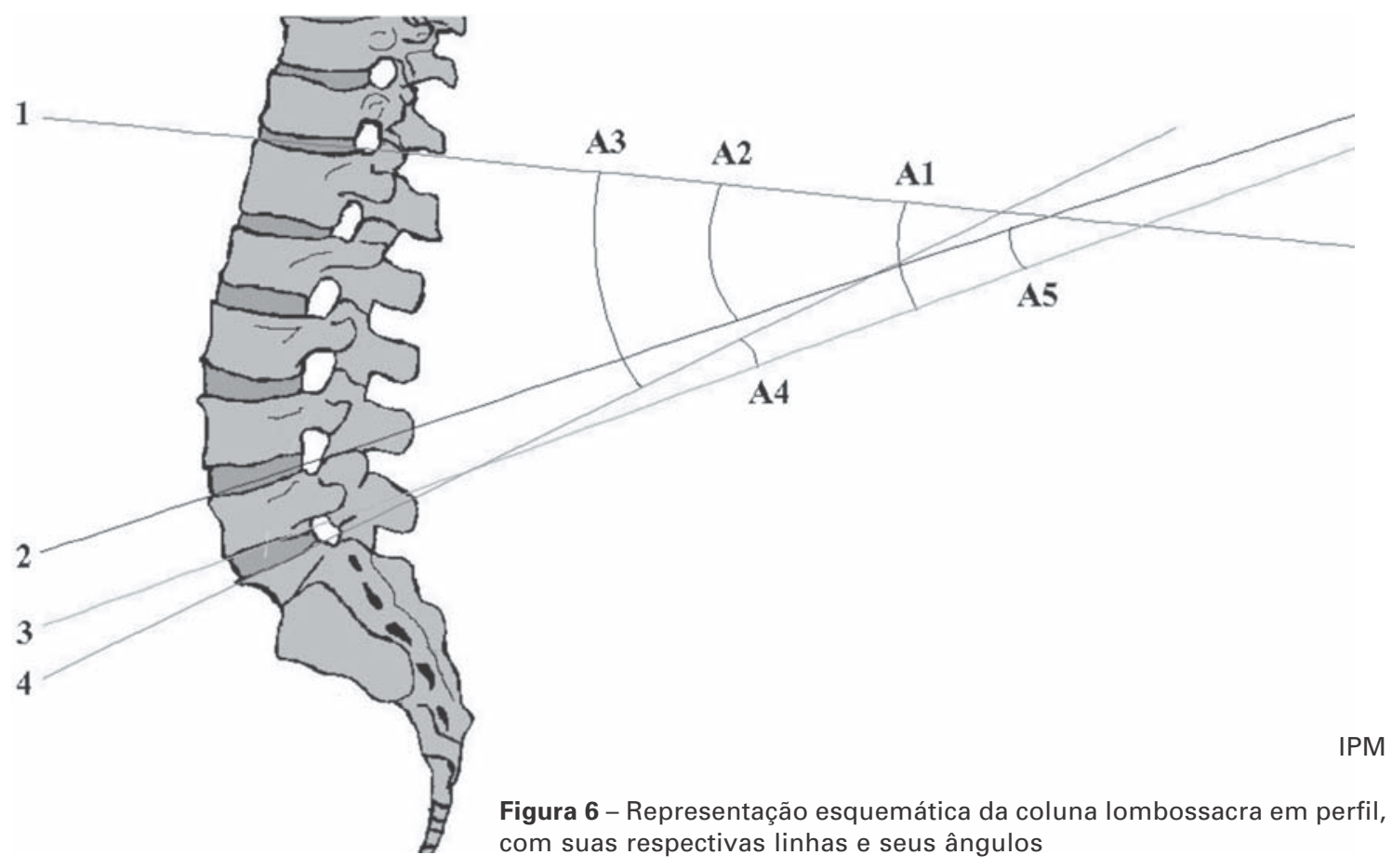

TABE

\begin{tabular}{|c|c|c|c|c|c|c|c|c|c|}
\hline \multirow[t]{2}{*}{ Grupo } & \multirow[t]{2}{*}{ Sexo } & \multirow[t]{2}{*}{ Casuística } & \multirow[t]{2}{*}{ Teste estatístico } & \multirow[t]{2}{*}{ Idade } & \multirow[t]{2}{*}{$\begin{array}{c}\text { Teste } \\
\text { abdominal } \\
\text { I }\end{array}$} & \multirow[t]{2}{*}{$\begin{array}{c}\text { Teste } \\
\text { abdominal } \\
\text { II }\end{array}$} & \multicolumn{3}{|c|}{$\begin{array}{c}\text { Lordose lombar superior } \\
\text { Witse e Winter } \\
\text { L1 topo a L5 topo }\end{array}$} \\
\hline & & & & & & & Neutro & Flexão & Mobilidade \\
\hline \multirow{3}{*}{ I } & Masculino & 29 & $\begin{array}{l}\text { Média aritmética } \\
\text { Desvio-padrão }\end{array}$ & $\begin{array}{l}9 \\
5\end{array}$ & $\begin{array}{r}100 \% \\
0 \%\end{array}$ & $\begin{array}{r}100 \% \\
0 \%\end{array}$ & $\begin{array}{r}30,4 \\
7,1\end{array}$ & $\begin{array}{r}-19,1 \\
8,7\end{array}$ & $\begin{array}{r}49,6 \\
9,1\end{array}$ \\
\hline & Feminino & 21 & $\begin{array}{l}\text { Média aritmética } \\
\text { Desvio-padrão }\end{array}$ & $\begin{array}{r}11 \\
5\end{array}$ & $\begin{array}{r}100 \% \\
0 \%\end{array}$ & $\begin{array}{l}90 \% \\
30 \%\end{array}$ & $\begin{array}{r}37,3 \\
7,6\end{array}$ & $\begin{array}{r}-18,3 \\
7,6\end{array}$ & $\begin{array}{l}55,6 \\
10,7\end{array}$ \\
\hline & Total & 50 & $\begin{array}{l}\text { Média aritmética } \\
\text { Desvio-padrão }\end{array}$ & $\begin{array}{r}10 \\
5\end{array}$ & $\begin{array}{r}100 \% \\
0 \%\end{array}$ & $\begin{array}{l}96 \% \\
20 \%\end{array}$ & $\begin{array}{c}33,3 \\
8\end{array}$ & $\begin{array}{r}-18,8 \\
8,2\end{array}$ & $\begin{array}{l}52,1 \\
10,2\end{array}$ \\
\hline \multirow{3}{*}{ II } & Masculino & 25 & $\begin{array}{l}\text { Média aritmética } \\
\text { Desvio-padrão }\end{array}$ & $\begin{array}{r}31 \\
7\end{array}$ & $\begin{array}{l}68 \% \\
48 \%\end{array}$ & $\begin{array}{l}48 \% \\
51 \%\end{array}$ & $\begin{array}{l}32,4 \\
10,6\end{array}$ & $\begin{array}{l}3,1 \\
6,4\end{array}$ & $\begin{array}{l}29,4 \\
12,1\end{array}$ \\
\hline & Feminino & 25 & $\begin{array}{l}\text { Média aritmética } \\
\text { Desvio-padrão }\end{array}$ & $\begin{array}{r}31 \\
6\end{array}$ & $\begin{array}{l}72 \% \\
46 \%\end{array}$ & $\begin{array}{l}44 \% \\
51 \%\end{array}$ & $\begin{array}{r}39,8 \\
9,2\end{array}$ & $\begin{array}{l}5,3 \\
4,3\end{array}$ & $\begin{array}{l}34,5 \\
10\end{array}$ \\
\hline & Total & 50 & $\begin{array}{l}\text { Média aritmética } \\
\text { Desvio-padrão }\end{array}$ & $\begin{array}{r}31 \\
6\end{array}$ & $\begin{array}{l}70 \% \\
46 \%\end{array}$ & $\begin{array}{l}46 \% \\
50 \%\end{array}$ & $\begin{array}{l}36,1 \\
10,5\end{array}$ & $\begin{array}{l}4,2 \\
5,5\end{array}$ & $\begin{array}{l}31,9 \\
11,3\end{array}$ \\
\hline \multirow{3}{*}{ III } & Masculino & 26 & $\begin{array}{l}\text { Média aritmética } \\
\text { Desvio-padrão }\end{array}$ & $\begin{array}{r}52 \\
6\end{array}$ & $\begin{array}{l}62 \% \\
50 \%\end{array}$ & $\begin{array}{l}27 \% \\
45 \%\end{array}$ & $\begin{array}{c}39,2 \\
6\end{array}$ & $\begin{array}{r}11,9 \\
6,7\end{array}$ & $\begin{array}{r}27,3 \\
7,9\end{array}$ \\
\hline & Feminino & 24 & $\begin{array}{l}\text { Média aritmética } \\
\text { Desvio-padrão }\end{array}$ & $\begin{array}{r}51 \\
6\end{array}$ & $\begin{array}{l}33 \% \\
48 \%\end{array}$ & $\begin{array}{r}4 \% \\
20 \%\end{array}$ & $\begin{array}{l}43,4 \\
7\end{array}$ & $\begin{array}{c}13,4 \\
6\end{array}$ & $\begin{array}{l}30 \\
8,7\end{array}$ \\
\hline & Total & 50 & $\begin{array}{l}\text { Média aritmética } \\
\text { Desvio-padrão }\end{array}$ & $\begin{array}{r}51 \\
6\end{array}$ & $\begin{array}{l}48 \% \\
50 \%\end{array}$ & $\begin{array}{l}16 \% \\
37 \%\end{array}$ & $\begin{array}{r}41,2 \\
6,8\end{array}$ & $\begin{array}{r}12,6 \\
6,4\end{array}$ & $\begin{array}{r}28,6 \\
8,3\end{array}$ \\
\hline
\end{tabular}

Fonte dos dados: Ambulatório de Ortopedia do HUCFF-UFRJ entre 1995-2005. 
(48\% dos homens e $44 \%$ das mulheres). No grupo III, para os testes I e II, os valores foram de $48 \%$ (62\% dos homens e 33\% das mulheres) e $16 \%$ (27\% dos homens e $4 \%$ das mulheres), respectivamente. As diferenças foram estatisticamente significantes entre os grupos. Entre os sexos não houve diferença apenas no teste I nos grupos I e II, sendo todas as outras comparações estatisticamente significantes.

Foram medidos os ângulos da lordose lombar superior (do topo de L1 ao topo de L5), com valor médio, no grupo I, de $33,3^{\circ}\left(30,4^{\circ}\right.$ nos homens e $37,3^{\circ}$ nas mulheres) na posição neutra e de $-18,8^{\circ}\left(-19,1^{\circ}\right.$ nos homens e $-18,3^{\circ}$ nas mulheres) em flexão máxima. Nos grupos II e III os valores foram, respectivamente, de $36,1^{\circ}\left(32,4^{\circ}\right.$ nos homens e $39,8^{\circ}$ nas mulheres) e $4,2^{\circ}\left(3,1^{\circ}\right.$ nos homens e $5,3^{\circ}$ nas mulheres) no II e $41,2^{\circ}\left(39,2^{\circ}\right.$ nos homens e $43,4^{\circ}$ nas mulheres) e $12,6^{\circ}$ $\left(11,9^{\circ}\right.$ nos homens e $13,4^{\circ}$ nas mulheres) no grupo III. As diferenças foram estatisticamente significativas entre os grupos. A média geral foi de $37^{\circ}$ na posição neutra e $-1^{\circ}$ na flexão máxima. Os resultados podem ser apreciados no gráfico 2 .

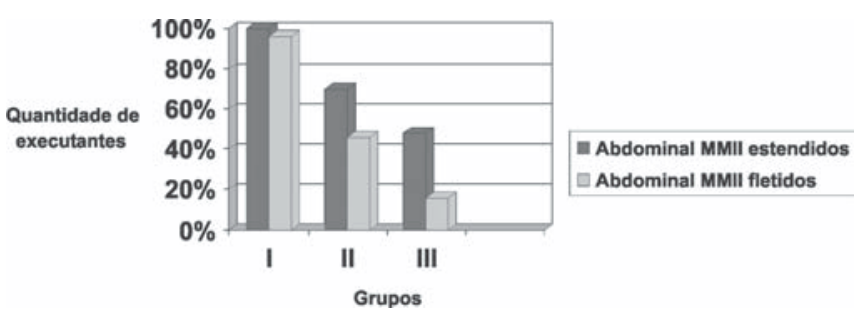

Gráfico 1 - Resultado do teste abdominal

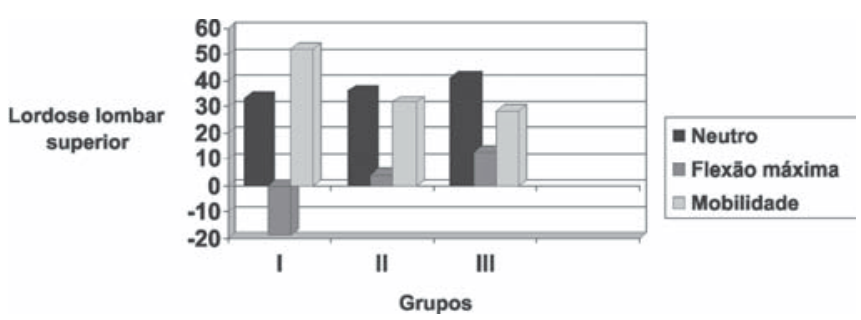

Gráfico 2 - Medida do ângulo da lordose lombar superior

\begin{tabular}{|c|c|c|c|c|c|c|c|c|c|c|c|}
\hline \multicolumn{3}{|c|}{$\begin{array}{l}\text { Lordose lombar inferior } \\
\text { Propst-Proctor e Bleck } \\
\text { L1 topo a L5 base }\end{array}$} & \multicolumn{3}{|c|}{$\begin{array}{l}\text { Lordose lombar total } \\
\text { Lordose lombossacra } \\
\text { L1 topo a S1 topo }\end{array}$} & \multicolumn{3}{|c|}{$\begin{array}{l}\text { Ângulo da articulação } \\
\text { lombossacra } \\
\text { L5 base a S1 topo }\end{array}$} & \multicolumn{3}{|c|}{$\begin{array}{l}\text { Acunhamento da } \\
5 \text { sértebra lombar } \\
\text { L5 topo a L5 base }\end{array}$} \\
\hline Neutro & Flexão & Mobilidade & Neutro & Flexão & Mobilidade & Neutro & Flexão & Mobilidade & Neutro & Flexão & Mobilidade \\
\hline 39,1 & $-12,4$ & 51,6 & 54,9 & $-12,9$ & 67,8 & 15,8 & 1 & 14,8 & 8,3 & 5,8 & 2,6 \\
\hline 7,4 & 8,9 & 8,6 & 8,9 & 7,7 & 8,8 & 5,8 & 5 & 6,1 & 3,9 & 3,9 & 6 \\
\hline 46 & $-12,3$ & 58,3 & 59,2 & $-11,7$ & 70,9 & 13 & 1,9 & 11,1 & 9 & 6 & 3 \\
\hline 7,8 & 9,1 & 11,2 & 9,1 & 8,9 & 10,1 & 6,4 & 6 & 8,7 & 3,4 & 4 & 3,9 \\
\hline 42 & $-12,4$ & 54,4 & 56,7 & $-12,4$ & 69,1 & 14,6 & 1,4 & 13,2 & 8,6 & 5,9 & 2,7 \\
\hline 8,2 & 8,9 & 10,2 & 9,1 & 8,1 & 9,4 & 6,2 & 5,4 & 7,5 & 3,7 & 3,9 & 5,2 \\
\hline 39 & 10 & 29 & 53,4 & 18,9 & 34,5 & 12,8 & 9 & 3,8 & 8,2 & 6,4 & 1,8 \\
\hline 12,5 & 5,7 & 13,3 & 9,7 & 6,2 & 13 & 5,6 & 4,4 & 3,9 & 2,5 & 2,5 & 2,6 \\
\hline 46,8 & 11,2 & 35,6 & 58,4 & 17,3 & 41,1 & 11,6 & 6,1 & 5,5 & 6,9 & 5,8 & 1,1 \\
\hline 9,2 & 4 & 10,2 & 10,7 & 5,5 & 11,9 & 4,6 & 4 & 4,2 & 2,7 & 2,6 & 1,8 \\
\hline 42,9 & 10,6 & 32,3 & 55,9 & 18,1 & 37,8 & 12,2 & 7,5 & 4,7 & 7,6 & 6,1 & 1,4 \\
\hline 11,5 & 4,9 & 12,2 & 10,4 & 5,9 & 12,8 & 5,1 & 4,4 & 4,1 & 2,7 & 2,6 & 2,2 \\
\hline 46,2 & 18,9 & 27,3 & 56 & 26 & 30 & 9,6 & 7,1 & 2,5 & 4,3 & 3,6 & 0,7 \\
\hline 5,1 & 7,8 & 6,9 & 5,8 & 7 & 6,7 & 3,2 & 2,7 & 1,8 & 1,6 & 1,5 & 0,9 \\
\hline 50 & 18,2 & 31,8 & 60,7 & 27 & 33,6 & 10,7 & 8,9 & 1,8 & 4,5 & 3,6 & 0,9 \\
\hline 5,6 & 5,4 & 7,3 & 5,9 & 5,6 & 7,3 & 2,6 & 2,3 & 1 & 2,1 & 1,6 & 1,2 \\
\hline 48 & 18,5 & 29,4 & 58,2 & 26,5 & 31,7 & 10,1 & 7,9 & 2,2 & 4,4 & 3,6 & 0,8 \\
\hline 5,6 & 6,7 & 7,4 & 6,2 & 6,3 & 7,1 & 3 & 2,7 & 1,5 & 1,8 & 1,6 & 1 \\
\hline
\end{tabular}




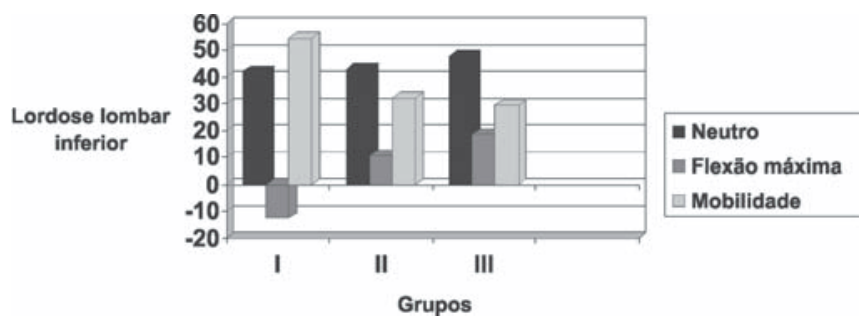

Gráfico 3 - Medida do ângulo da lordose lombar inferior

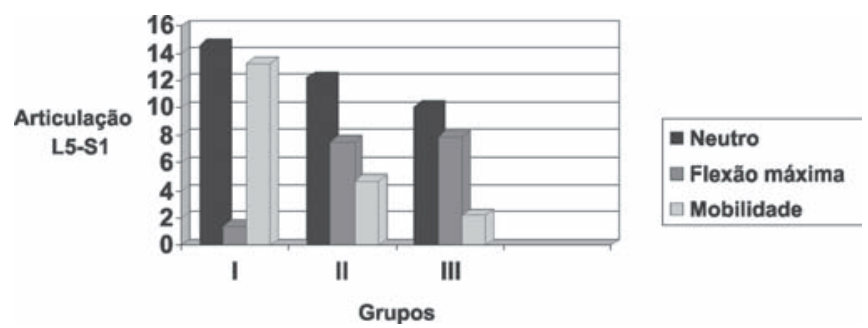

Gráfico 5 - Medida do ângulo da articulação lombossacral

O ângulo da lordose lombar inferior (do topo de L1 à base de L5) teve valor médio de $42^{\circ}\left(39,1^{\circ}\right.$ nos homens e $46^{\circ}$ nas mulheres) na posição neutra e de $12,4^{\circ}\left(-12,4^{\circ}\right.$ nos homens e $-12,3^{\circ}$ nas mulheres) em flexão máxima no grupo I; de $42,9^{\circ}\left(39^{\circ}\right.$ nos homens e $46,8^{\circ}$ nas mulheres) e $10,6^{\circ}\left(10^{\circ}\right.$ nos homens e $11,2^{\circ}$ nas mulheres) no grupo II e $48^{\circ}\left(46,2^{\circ}\right.$ nos homens e $50^{\circ}$ nas mulheres $)$ e $18,5^{\circ}\left(18,9^{\circ}\right.$ nos homens e $18,2^{\circ}$ nas mulheres) no grupo III. As diferenças não foram estatisticamente significantes entre os grupos I e II, mas foram entre o grupo III e os grupos I e II. A média geral foi de $44^{\circ}$ na posição neutra e $6^{\circ}$ na flexão máxima. Os resultados podem ser vistos no gráfico 3 .

O ângulo da lordose lombossacra ou lordose total (do topo de $\mathrm{L} 1$ ao topo de $\mathrm{S} 1$ ) teve média de $56,7^{\circ}$ $\left(54,9^{\circ}\right.$ nos homens e $59,2^{\circ}$ nas mulheres) na posição neutra e $-12,4^{\circ}\left(-12,9^{\circ}\right.$ nos homens e $-11,7^{\circ}$ nas mulheres) em flexão máxima no grupo I; $55,9^{\circ}\left(53,4^{\circ}\right.$ nos homens e $58,4^{\circ}$ nas mulheres $)$ e $18,1^{\circ}\left(18,9^{\circ}\right.$ nos homens e $17,3^{\circ}$ nas mulheres) no grupo II; e $58,2^{\circ}\left(56^{\circ}\right.$ nos homens e $60,7^{\circ}$ nas mulheres) e $26,5^{\circ}$ ( $26^{\circ}$ nos homens e $27^{\circ}$ nas mulheres) no grupo III. Não houve diferença significativa nas medições em neutro entre os grupos, mas o decréscimo da mobilidade foi significativo. A média geral foi de $57^{\circ}$ na posição neutra e

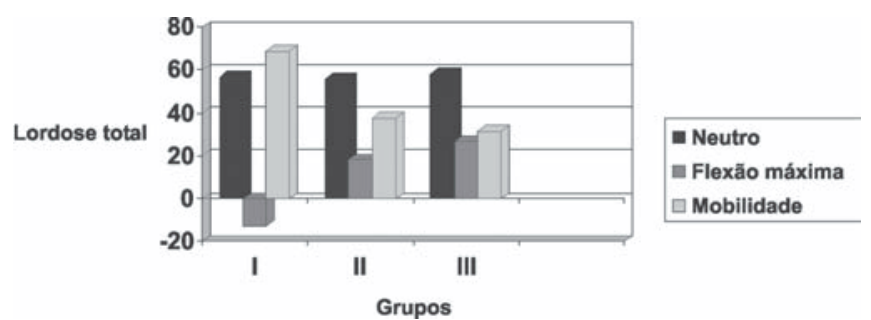

Gráfico 4 - Medida do ângulo da lordose total

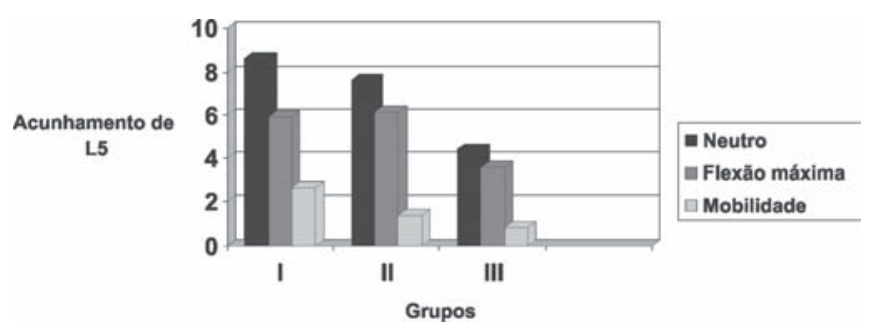

Gráfico 6 - Medida do ângulo da cunha de L5

$11^{\circ}$ na flexão máxima. Os resultados podem ser vistos no gráfico 4.

O ângulo da articulação lombossacral (da base de L5 ao topo de S1) também foi medido, com resultados médios na posição neutra e em flexão máxima de $14,6^{\circ}$ $\left(15,8^{\circ}\right.$ nos homens e $13^{\circ}$ nas mulheres) e $1,4^{\circ}\left(1^{\circ}\right.$ nos homens e $1,9^{\circ}$ nas mulheres), respectivamente, no grupo I; $12,2^{\circ}\left(12,8^{\circ}\right.$ nos homens e $11,6^{\circ}$ nas mulheres $)$ e $7,5^{\circ}\left(9^{\circ}\right.$ nos homens e $6,1^{\circ}$ nas mulheres) no grupo II; e $10,1^{\circ}\left(9,6^{\circ}\right.$ nos homens e $10,7^{\circ}$ nas mulheres) e $7,9^{\circ}$ $\left(7,1^{\circ}\right.$ nos homens e $8,9^{\circ}$ nas mulheres) no grupo III. As diferenças foram estatisticamente significativas entre os grupos. Os resultados podem ser vistos no gráfico 5 .

O ângulo da cunha de L5 (do topo de L5 à base de L5) quando medido na posição neutra e em flexão máxima apresentou médias de: $8,6^{\circ}\left(8,3^{\circ}\right.$ nos homens e $9^{\circ}$ nas mulheres $)$ e $5,9^{\circ}\left(5,8^{\circ}\right.$ nos homens e $6^{\circ}$ nas mulheres) no grupo I; $7,6^{\circ}\left(8,2^{\circ}\right.$ nos homens e $6,9^{\circ}$ nas mulheres) e $6,1^{\circ}\left(6,4^{\circ}\right.$ nos homens e $5,8^{\circ}$ nas mulheres) no grupo II; e de $4,4^{\circ}\left(4,3^{\circ}\right.$ nos homens e $4,5^{\circ}$ nas mulheres) e $3,6^{\circ}$ (3, $6^{\circ}$ nos homens e $3,6^{\circ}$ nas mulheres) no grupo III. Não houve diferença estatística entre os grupos I e II, mas entre o grupo III e os grupos I e II. Os resultados podem ser vistos no gráfico 6. 


\section{DISCUSSÃO}

A análise dos resultados mostrou que existe um processo de estruturação da lordose lombar e diminuição da sua flexibilidade com o aumento da idade.

A capacidade de realizar os testes abdominais apresentou um padrão constante de queda com o aumento da faixa etária, partindo-se de uma taxa de 100 no grupo I. A taxa de realização do teste II, com o isolamento da musculatura abdominal, foi sempre inferior à do teste I, com o auxílio dos flexores da pelve. Só houve diferença significativa em relação ao sexo no grupo mais velho, no qual o sexo masculino apresentou maior capacidade de realização dos dois testes. Nossos resultados mostram a musculatura antilordogênica abdominal mais fraca e se deteriorando mais rapidamente que o iliopsoas lordogênico.

Charrière descreveu dois grupos musculares distintos atuantes sobre a coluna lombar. Um grupo lordogênico, anteversor da pelve, e outro antilordogênico, retroversor da pelve (reto abdominal, oblíquo, glúteos e isquiotibiais) $)^{(3)}$. Koyama observou que o reto abdominal era mais fraco que o psoas em pacientes com lombalgia crônica e identificou insuficiência dos abdominais, com predomínio do psoas-ilíaco, em substituição à ação dos músculos da parede abdominal ${ }^{(4)}$. Essa combinação agravaria o efeito lordogênico, aumentando a pressão nas articulações interfacetárias, principalmente nos níveis L4-L5 e L5-S1. Tais fatores seriam decisivos na gênese do processo de estruturação da lordose lombar, limitando o movimento de flexão, como descrito por Charrière ${ }^{(3)}$.

Von Lackum observou que a estruturação da coluna em lordose se iniciaria a partir dos 40 anos de idade, determinando aumento da lordose e redução da amplitude de movimento de flexo-extensão lombar, como resultado do encurtamento da musculatura paravertebral, levando à posição lordótica mais fixa por falência da musculatura retroversora, antilordogênica (retos abdominais e oblíquos) $)^{(5)}$.

Observamos que a lordose lombar aumentou, tanto quando medida na base ou no topo de L5, e sua mobilidade diminuiu com o aumento da idade. Em todas as faixas etárias, o sexo feminino apresentou valores maiores para a lordose lombar, mas a mobilidade foi semelhante à do sexo masculino. Propst-Proctor et al, Wright et al, e Stagnara et al não encontraram diferenças significativas relativas ao $\mathrm{sexo}^{(1,6-7)}$. Koyama ressaltou maior prevalência de lombalgia no sexo feminino, observando graus maiores de lordose lombar em mulheres $^{(4)}$. Van Herp et al, analisando 100 indivíduos (50 homens e 50 mulheres), evidenciaram que o sexo feminino apresentava maior flexibilidade lombar em todas as faixas etárias estudadas ${ }^{(8)}$.

Detectamos que o ângulo de lordose medida do topo de L1 ao topo de L5 teve crescimento do valor angular médio nos três grupos etários avaliados, tanto em neutro ortostático como em flexão máxima, passando, respectivamente, de $33,3^{\circ}$ e $-18,8^{\circ}$ no grupo I, para $36,1^{\circ}$ e $4,2^{\circ}$ no grupo II e $41,2^{\circ}$ e $12,6^{\circ}$ no grupo III. Isso traduziu diminuição de $52,1^{\circ}$ da mobilidade verificada na faixa de 0 a 20 anos, para $31,9^{\circ}$ na de 21 a 40 anos, e $28,6^{\circ}$ na de 41 a 60 anos. Tais achados vão ao encontro dos de Wright et al que, utilizando o mesmo parâmetro para analisar o ângulo da lordose lombar em crianças de até 18 anos, encontraram valor médio de $35^{\circ}$, semelhante aos $33,3^{\circ}$ deste estudo $^{(6)}$.

O valor médio do ângulo da lordose, do topo de L1 à base de L5, encontrado por Propst-Proctor et al, analisando um grupo de crianças de até 13 anos, foi de $40^{\circ}$, em ortostatismo ${ }^{(1)}$. Tal média foi semelhante aos $42^{\circ}$ encontrados neste estudo na faixa etária de 0 a 20 anos, em que o valor médio em flexão máxima foi de $-12,4^{\circ}$, com média de $54,4^{\circ}$ de mobilidade da coluna lombar. Essas médias foram se alterando nas faixas etárias superiores, sendo de $42,9^{\circ}$ e $10,6^{\circ}$ na de 21 a 40 anos, com $32,3^{\circ}$ de mobilidade e de $48^{\circ}$ e $18,5^{\circ}$ na de 41 a 60 anos, com $29,4^{\circ}$ de mobilidade. Nota-se que, com o aumento da faixa etária, houve progressão da lordose lombar, com diminuição da mobilidade desse segmento da coluna vertebral.

O ângulo da articulação lombossacral, medido da base de L5 ao topo de S1, apresentou diminuição progressiva com a idade, bem como perda de mobilidade. Não foram observadas diferenças significativas em relação ao sexo. O resultado médio na posição neutra foi de $14,6^{\circ}$ no grupo I, em concordância com os $12^{\circ}$ relata- 
dos por Propst-Proctor et al e com os $14^{\circ}$ encontrados por Wright e Bell ${ }^{(1,6)}$. Em nossa pesquisa, a média em flexão nessa faixa foi de $1,4^{\circ}$, determinando mobilidade, nessa articulação, de $13,2^{\circ}$. No grupo II, as médias foram de $12,2^{\circ}$ e $7,5^{\circ}$, com mobilidade de $4,7^{\circ}$, enquanto no grupo III, de $10,1^{\circ}$ e $7,9^{\circ}$, com mobilidade média de $2,2^{\circ}$. Sendo essa articulação essencialmente lordótica, não faz parte do conjunto que sofre inversão no sentido da cifose durante o movimento de flexão.

Observamos que a cunha de L5 apresentou diminuição constante com o aumento da idade, mas nenhuma diferença significativa em relação ao sexo. $\mathrm{O}$ ângulo de acunhamento da quinta vértebra lombar (do topo de L5 à base de L5) teve mínima variação na posição neutra e em flexão máxima, com valores médios no grupo I de $8,6^{\circ}$ e $5,9^{\circ}$, de $7,6^{\circ}$ e $6,1^{\circ}$ no grupo II e de $4,4^{\circ}$ e $3,6^{\circ}$ no grupo III. Os valores não mostram inversão da lordose lombar para cifose, indicando que L5 é uma vértebra lordogênica, sem participação na inversão da lordose lombar quando em flexão máxima, independente da faixa etária. A lordose total, medida do topo de L1 ao topo de $\mathrm{S} 1$, ao contrário, permaneceu praticamente inalterada nas três faixas etárias, mas sendo sempre maior no sexo feminino. Sua mobilidade, no entanto, diminuiu consistentemente com o aumento da idade, sendo também sempre maior no sexo feminino. Na faixa de 0 a 20 anos, foi verificada média de $56,7^{\circ}$ na posição neutra e de $-12,4^{\circ}$ em flexão máxima, com média da mobilidade lombar de $69,1^{\circ}$. Araújo et al, analisando crianças de cinco a 10 anos, apresentaram média da lordose lombar, apenas na posição ortostática, mas com esses mesmos parâmetros, de 53,01 ${ }^{\circ(9)}$.

No grupo II, as médias foram de $55,9^{\circ}$ e $18,1^{\circ}$, com mobilidade de $37,8^{\circ}$, não tendo havido inversão da lordose lombar quando em flexão máxima. Resultados semelhantes apresentaram Stagnara et al que, estudando indivíduos entre 20 e 29 anos e medindo o ângulo entre as vértebras mais inclinadas da curva, acharam média de $50^{\circ}{ }^{(7)}$. Fernand e Fox ${ }^{(10)}$, medindo o ângulo entre L2 e S1, obtiveram média de $45,05^{\circ}$.

No grupo III, as médias, por nós identificadas, foram de $58,2^{\circ} \mathrm{em}$ posição ortostática e de $26,5^{\circ} \mathrm{em}$ flexão máxima, determinando média de $31,7^{\circ}$ na mobilidade da coluna lombar. A média de todas as faixas etárias foi de $57^{\circ}$. Jackson et al, utilizando os mesmos parâmetros, encontraram valor médio de $62,1^{\circ}$ em 50 indivíduos da faixa etária entre 20 e $65 \operatorname{anos}^{(11)}$. Medindo entre o topo de T12 e o topo de S1, em 75 indivíduos numa faixa etária entre 20 e 63 anos, Jackson e Hales encontraram média de $62,5^{\circ}(12)$. Vaz et al, avaliando 100 pacientes entre 20 e 45 anos e medindo a lordose entre as vértebras mais inclinadas da curva, encontraram média de $46,5^{\circ}(13)$. Korovessis et al, em estudo de 99 pacientes, entre 20 e 79 anos, acharam média de lordose lombar de $83^{\circ}$, medida entre o topo de $\mathrm{T} 12$ e o topo de $\mathrm{S} 1^{(14)}$.

É importante ressaltar que a diferença de mobilidade entre os grupos I e III foi de $37,4^{\circ}$, ou seja, a perda foi significativa. Outra evidência foi a perda progressiva da capacidade de movimentar a coluna lombar no sentido da cifose nos grupos II e III, quando comparados com o que ocorre na faixa etária de 0 a 20 anos, em todos os parâmetros estudados.

A perda de qualidade da ação dos músculos antilordogênicos, a redução da mobilidade da coluna lombar que ocorreu com o avanço da idade, somada à postura lordótica da vértebra de L5 e da articulação L5-S1, seriam as causas do aumento da pressão nas articulações interfacetárias, principalmente nos níveis L4-L5 e L5-S1, propiciando o desenvolvimento da síndrome facetária.

Os valores médios encontrados no presente estudo mostraram um progressivo processo de estruturação da lordose lombar, limitando a flexão com o aumento da faixa etária.

Fazzi e Araújo enfatizaram como causa de lombalgia o comprometimento vertebral posterior devido, principalmente, ao processo degenerativo e falência da musculatura retroversora ${ }^{(15)}$. Lewin ressaltou a influência da estruturação da lordose como causa de aumento da pressão interfacetária, determinando lombalgia persistente ${ }^{(16)}$. Koyama demonstrou existir média de $20^{\circ}$ de mobilidade lombar, medida entre a lordose em flexão e a lordose em extensão, em pacientes portadores de lombalgia ${ }^{(4)}$.

A constatação desses dados evidencia a importância da criação de um programa de saúde com objetivo de preservar a força muscular antilordogênica (exercí- 
cios abdominais) e a mobilidade em flexão (alongamentos dos músculos paravertebrais) da coluna lombar, fatores importantes na profilaxia de lombalgia crônica, caracterizada como artrose interapofisária.

\section{CONCLUSÃO}

Não houve diferença entre as médias das lordoses de L1 a S1, perfazendo média de $57^{\circ}$ nos três grupos etários e, portanto, não ocorreu acentuação da lordose com a idade.

Com a progressão da idade ocorreu a redução da amplitude de movimento de flexão lombar de L1 a S1, passando de $69,1^{\circ}$ nos jovens para $31,7^{\circ}$ nos idosos.

A eficiência da musculatura abdominal, como medida pela capacidade de executar os testes abdominais, diminuiu com a idade, sendo observado que $96 \%$ dos jovens conseguiam executar o exercício abdominal com os quadris fletidos, enquanto apenas $18 \%$ dos idosos o faziam. No teste para os psoas-ilíacos, $100 \%$ dos jovens o executaram, contra $44 \%$ dos idosos somente. Esses fatos demonstram que há, com o correr dos anos, progressiva falência da musculatura flexora abdominal, eminentemente cifogênica, e substituição pelos psoas-ilíacos, lordogênicos.

\section{AGRADECIMENTOS}

Agradecimentos à Dra. Isabela Peixoto de Mello, pelos desenhos.

\section{REFERÊNCIAS}

1. Propst-Proctor SL, Bleck EE. Radiographic determination of lordosis and kyphosis in normal and scoliotic children. J Pediatr Orthop. 1983;3(3):344-6.

2. Wiltse LL, Winter RB. Terminology and measurement of spondylolisthesis. J Bone Joint Surg Am. 1983;65(6):768-72.

3. Charrière L. Kinesiterapia en el tratamiento de las algias vertebrales. 2a ed. Barcelona: Toray-Masson; 1974. p. 63-119.
4. Koyama PS. A importância da hiperlordose na lombalgia crônica [tese]. Rio de Janeiro: Faculdade de Medicina da Universidade Federal do Rio de Janeiro; 1993.

5. Von Lackum HL. The lumbosacral region: an anatomic study and some clinical observations. J Am Med Assoc. 1924;82: 1109-14.

6. Wright JG, Bell D. Lumbosacral joint angles in children. J Pediatr Orthop. 1991;11(6):748-51. Comment in: J Pediatr Orthop. 1992;12(5):676-7.

7. Stagnara P, De Mauroy JC, Dran G, Gonon GP, Costanzo G, Dimnet J, Pasquet A. Reciprocal angulation of vertebral bodies in a sagittal plane: approach to references for the evaluation of kyphosis and lordosis. Spine. 1982;7(4):335-42.

8. Van Herp G, Rowe P, Salter P, Paul JP. Three-dimensional lumbar spinal kinematics: a study of range of movement in 100 healthy subjects aged 20 to 60+ years. Rheumatology (Oxford). 2000;39(12):1337-40.

9. Araújo FRC, Fazzi A. Valor do ângulo da lordose lombar e do ângulo lombossacro na criança. Rev Bras Ortop. 1988;23(9):26571.

10. Fernand R, Fox DE. Evaluation of lumbar lordosis. A prospective and retrospective study. Spine. 1985;10(9):799-803.

11. Jackson RP, Peterson MD, McManus AC, Hales C. Compensatory spinopelvic balance over the hip axis and better reliability in measuring lordosis to the pelvic radius on standing lateral radiographs of adult volunteers and patients. Spine. 1988;23(16):1750-67.

12. Jackson RP, Hales C. Congruent spinopelvic alignment on standing lateral radiographs of adult volunteers. Spine. 2000; 25(21):2808-15.

13. Vaz G, Roussouly P, Berthonnaud E, Dimnet J. Sagittal morphology and equilibrium of pelvis and spine. Eur Spine J. 2002;11(1):80-7.

14. Korovessis PG, Stamatakis MV, Baikousis AG. Reciprocal angulation of vertebral bodies in the sagittal plane in an asymptomatic Greek population. Spine. 1998;23(6):700-4; discussion 704-5.

15. Fazzi A, Araújo FRC. Importância do ângulo lombossacro na síndrome do compartimento posterior. Rev Hosp Clin Fac Med Univ São Paulo. 1986;41(2):80-3.

16. Lewin T. Osteoarthritis in lumbar synovial joints. A morphologic study. Acta Orthop Scand Suppl. 1964;Suppl 73: $1-112$. 